\title{
Experimental Heat Transfer Study of Endwall in a Linear Cascade with IR Thermography
}

\author{
Borja Rojo $^{1, \mathrm{a}}$, Carlos Jimenez ${ }^{1, \mathrm{~b}}$, and Valery Chernoray ${ }^{1, \mathrm{c}}$ \\ ${ }^{1}$ Chalmers University of Technology, Department of Applied Mechanics, 41296 Göteborg, Sweden
}

\begin{abstract}
This abstract presents an endwall heat transfer experimental data of air flow going through outlet guide vanes (OGVs) situated in a low speed linear cascade. The measurement technique for this experiment was infrared thermography. In order to calculate the heat transfer coefficient (HTC) on the endwall, it has been used an instrumented window with a controlled constant temperature in one side of a 5 millimeter Plexiglass in order to generate high temperature gradients and, therefore, by measuring the surface temperature one the other side of the Plexiglass, it is calculated the HTC. Due to the fact that Plexiglass material has not good optical properties at infrared spectrum, it has been used a thin layer of black paint (10-12 $\mu \mathrm{m})$ which has high emissivity ( 0.973 ) in the range of temperature that we are working. The Reynolds number for this experiment is 300000 in on and off-design configuration of the OGVs (on-design $25^{\circ}$ and off-design cases are $40^{\circ}$ and $-25^{\circ}$ incident angle). Furthermore, the on-design case is run at two different Reynolds number, 300000 and 450000. During this experiments it can be seen how changing the inlet angle to the OGVs produces significant differences on the heat transfer along the endwall. The main objective for this investigation is to study the heat transfer along the endwall of a linear cascade so that it would be a well-defined test case for CFD validation.
\end{abstract}

\section{Introduction}

Demands from industry on improving the efficiency in all kind of energy systems, including aero engines, are leading to focus on the research in more efficient propulsion systems. There are several ways to increase the energy efficiency in jet engines, i.e. increasing by-pass ratio, raising the combustion temperature, decreasing the weight of any component in the aircraft etc.

OGVs are located at the outlet of the last stage of a low pressure turbine (LPT). The main purpose of adding this component to an aero jet engine is to connect the external casing and the core of these engines. The OGVs have to be able to carry all the loads from the internal to the external structure. Furthermore, through them it is possible to provide access for pipes and electronics from the outer case to the shaft of the gas turbine. In addition to the structural and connecting functions, the OGVs also have an important aerodynamic role in a gas turbine engine due to the fact that they eliminate the swirl that comes from the LPT situated upstream. The flow around the OGVs is complex, there are secondary flows involved, boundary layer development and risk of flow separation as well. The design parameters that are required, from an aerodynamic point of view, are the minimization of the pressure drop and the capability of withstand flow separation. Moreover, the prediction of flow separation and heat transfer becomes more complicated when the OGV has an inlet incidence angle far the on-design operation condition. Even though there has been performed numerous investigations on endwall heat

\footnotetext{
a e-mail: borja.rojo@chalmers.se

b e-mail: jcarlos@chalmers.se

c e-mail: valery.chernoray@chalmers.se
}

transfer blades ([1]-[6]), very little experimental information is available in the field of the OGVs.

This paper is focused in the study of the heat transfer on the surface of a vane and endwalls situated in a lowspeed linear cascade. The heat transfer study performed can help to understand better the complex heat transfer mechanisms involved in this case, and hence, the information can be used for CFD validation purposes. Knowing the heat transfer mechanisms involved can lead to predict accurately the maximum temperature or thermal load on the surface of this component, and therefore, estimate the cooling if needed or select a lighter material that can handle the predicted thermal loads without melting or being sensitive to creep. This work is a continuation of previous investigation in a large-scale linear cascade performed at Chalmers University of Technology [7],[8]. An additional motivation for this work is the comparison between the experimental results using two different heat transfer measurement techniques.

\section{Experimental Facility}

These experiments were performed in a low-speed linear cascade located in Chalmers University of Technology. The facility (see figure 1) consists of a centrifugal fan $(30 \mathrm{~kW})$, a wide angle diffuser, a settling chamber where the outlet flow has not big wakes which were generated on the centrifugal fan, a two-dimensional contraction chamber, an inlet-section and a test section with boundary-layer suction. The test section is composed by 2 parallel discs where the inner discs constitute the upper and lower endwalls of the OGVs (for more details see [9]).

The working area has a cross section of 240x1200 mm. In the test section there are located $4 \mathrm{OGVs}$ and the pitch 


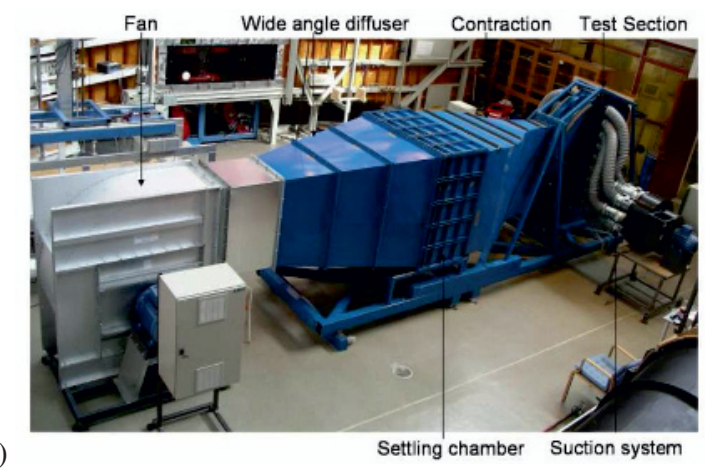

a)

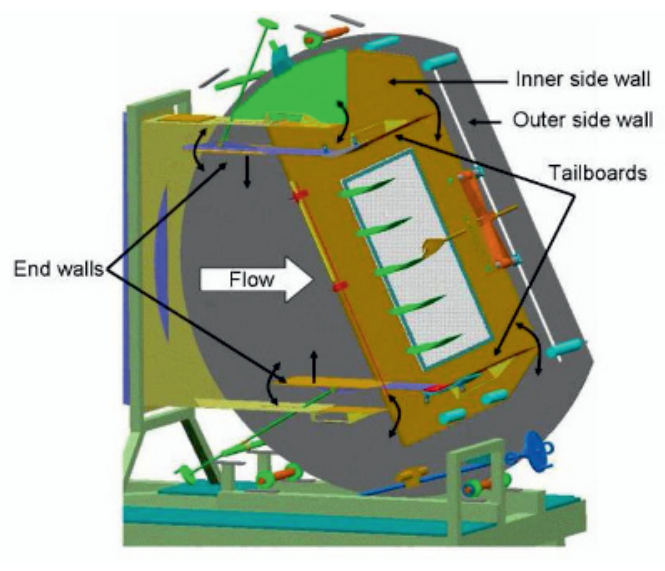

Fig. 1. a) Linear cascade sections. b) Test section. [9]

between them is $240 \mathrm{~mm}$. There is a vane which is instrumented for heat transfer measurements and 3 more made of plastic material. In order to control the periodicity in the test section, there are 2 tail-boards attached to the topmost and bottommost vanes. It has been tested the periodicity by measuring the static pressure on the vanes and by checking the wake profiles downstream the trailing edge of the vanes. The flow measurement procedure is explained in detail in [10]. The 4 OGVs are bolt to a Plexiglass wall. Furthermore, the level of the incoming flow turbulence intensity was adjusted by a turbulence grid which is located 700 $\mathrm{mm}$ upstream of the cascade. The OGVs are a $2 \mathrm{D}$ profile which is extended in the span direction.

\section{Theoretical Model}

For measuring the HTC on the linear cascade endwall, an instrumented window has been designed. In order to be able to measure the HTC, it is measured the temperature drop between two surfaces of the same body (in this case, a Plexiglass flat plate). Figure 2 shows the theoretical model that is used to explain the HTC measurement technique. All the terms can be calculated as:

$$
\begin{aligned}
& q_{c d}^{\prime \prime}=\frac{k}{t}\left(T_{a l}-T_{\text {plexi }}\right) \quad \text { Conduction heat flux } \\
& q_{c v}^{\prime \prime}=h\left(T_{\text {plexi }}-T_{\text {air }}\right) \quad \text { Convection heat flux }
\end{aligned}
$$

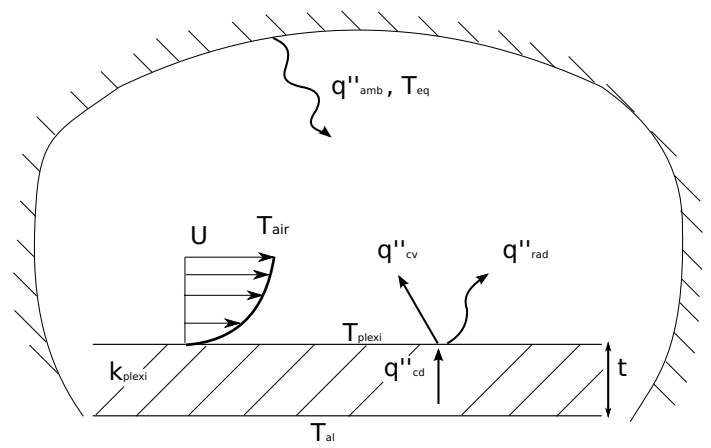

Fig. 2. Theoretical model for the HTC measurement.[11]

$$
q_{\text {rad }}^{\prime \prime} \simeq \epsilon \sigma T_{\text {plexi }}^{4}-\sigma T_{e q}^{4} \quad \text { Net radiation heat flux }
$$

Where $k$ is thermal conductivity of the Plexiglass which is $0.2 \mathrm{~W} \mathrm{~m}^{-2} \mathrm{~K}^{-1}, \mathrm{t}$ is the thickness of Plexiglass which is $5 \mathrm{~mm}, T_{a l}$ is the temperature on the interface between the Plexiglass and the aluminium (see figure 3 ) which is heated by 4 flat heaters, $T_{\text {plexi }}$ is the temperature on the external surface which is measured with the IR-camera, $T_{\text {air }}$ is the temperature of the air flow through the linear cascade far enough from the heated endwall, $\epsilon$ is the thermal emissivity of the surface of the Plexiglass which has been painted using the Nextel Vetel-Coating 811-21 from Mankiewicz Gebr. \& Co and its value is constant in our range of temperatures (0.973), $\sigma$ is the Boltzmann's constant $\left(5.67 \cdot 10^{-8} \mathrm{~W} \mathrm{~m}^{-2} \mathrm{~K}^{-4}\right)$ and $T_{e q}$ is the equivalent ambient temperature which is used to estimate the heat losses via radiation to the surroundings of the endwall. In this case, the convective heat transfer coefficient is high enough so that all the surroundings are at the air temperature. Furthermore, it has been measure with an IR-camera the empirical value of this parameter. Not taking into account this term can lead to an additional error of 3 to $10 \%$ (depending on the wall temperature in each point).

$$
q_{c d}^{\prime \prime}=q_{c v}^{\prime \prime}+q_{r a d}^{\prime \prime}
$$

Afterwards, doing energy balance (eq. 4) it can be obtained the measured HTC or $h$ (eq. 5).

$$
H T C=\frac{\frac{k}{t}\left(T_{a l}-T_{\text {plexi }}\right)-\left(\epsilon \sigma T_{\text {plexi }}^{4}-\sigma T_{e q}^{4}\right)}{T_{\text {plexi }}-T_{\text {air }}}
$$

\section{Experimental Setup}

\subsection{Instrumented Window}

As it is described in section 3, an instrumented window has been designed for obtaining the heat transfer on an endwall situated in a low-speed linear cascade. In order to design this window, it had to be taken into account several issues that affect the accuracy of the heat transfer measurement.

First of all, the Plexiglass plate which is situated in between the air flow and the aluminium plate has to be attached to the aluminium plate so that there is no air gap (100 $\mu \mathrm{m}$ air gap leads to $1.5^{\circ} \mathrm{C}$ error in the measurement). 

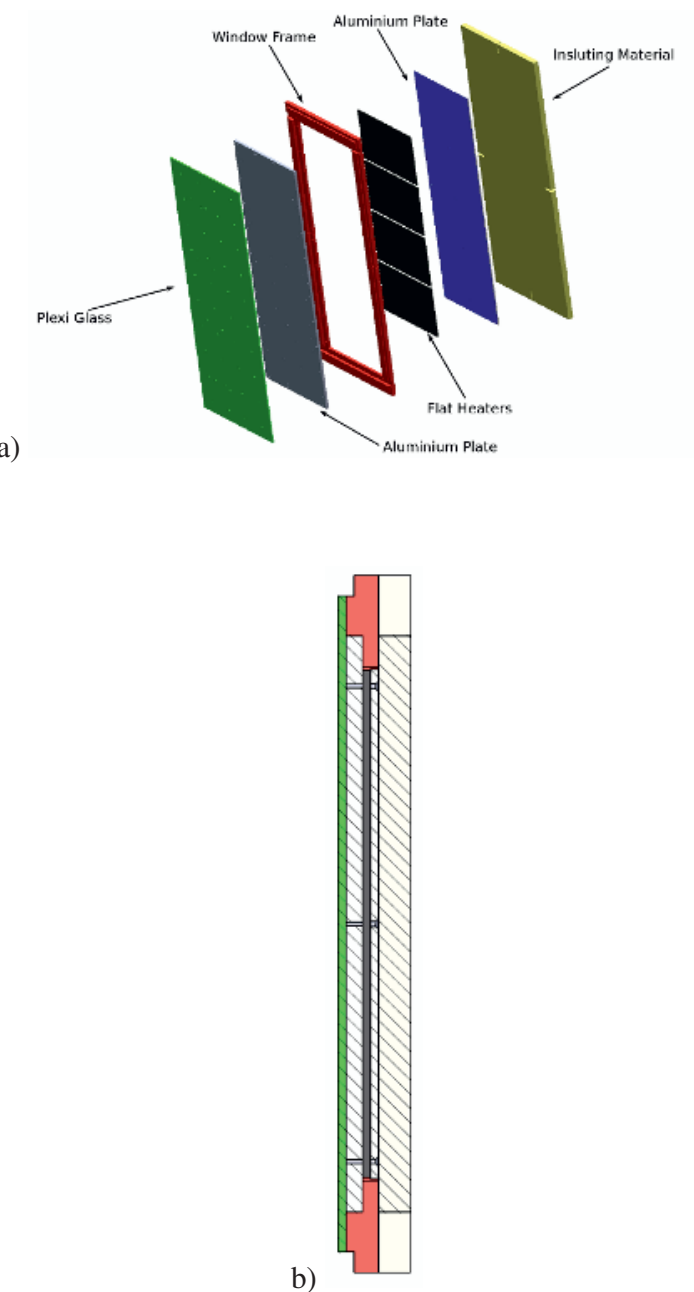

Fig. 3. a) Exploded view of the instrumented window. b) Cross section view.

Therefore, it is critical to have a good surface contact between this two flat plates. Then, the Plexiglass plate is attached to the aluminium plate bolting them with plastic screws. The reason for using plastic screws is that they have almost the same thermal conductivity as the Plexiglass and hence, they would not change the HTC in the surroundings of these screws.

Furthermore, the window needs to be isolated from the steel frame where it stands on the linear cascade. If the aluminium plate touches this steel frame, most of the heat would go through the steel plate to the air flow and therefore, it would not be any temperature gradient on the Plexiglass. In order to isolate the aluminium from the steel plate, a plastic frame has been installed on the instrumented window.

In addition, it is added in the back of the four heaters an aluminium plate. This plate has 2 functions. The first one is that it can be used to improve the contact between the heaters and the aluminium plate that provides the uniform temperature on the back of the Plexiglass. The second one is that it helps to have a more uniform temperature distribution in the window.

Finally, the insulating material situated on the back of the last aluminium plate adds more uniformity to the temperature distribution inside the instrumented window.
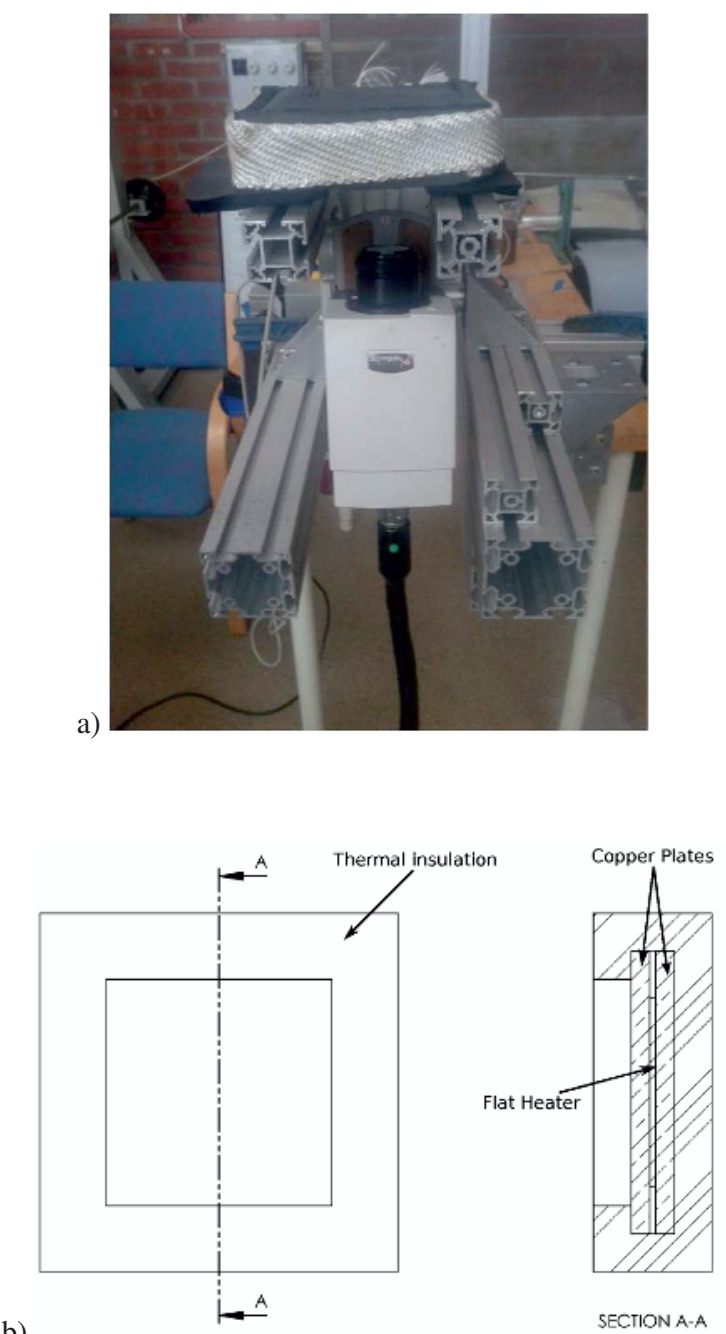

Fig. 4. a) Picture of the calibration process of the IR-camera. b) Drawing of the calibration tool.

\subsection{IR-Camera}

In order to measure the temperature distribution on the end-wall and further compute the HTC, an infrared camera is used. This is a MWIR Phoenix Camera System with a resolution of $320 \times 256$. This camera has been used in previous experiments in a large-scale low-speed annular cascade rig (see [12], [13]). Before the experiment starts, the camera must be calibrated and a non-uniformity correction is applied. In order to perform this set up for the IRcamera, it is needed to provide a uniform temperature over a surface. For this purpose, a calibration tool has been developed in our lab.

As it can be seen in figure 4, this calibration tool is composed by 2 copper plates, a flat heater and an insulating material around the plates except the exposed surface with an uniform temperature distribution. This surface is painted with the same paint that is used on the Plexiglass. This is a black paint (Nextel Velvet Coating 811-21 from Mankiewicz Gebr. \& Co.) which has a high emissivity of 0.973 and low reflectivity in the IR range up to an angle of $60^{\circ}$ between the IR-camera and the surface. A thermocouple is situated on the plate that the IR-camera looks at during the calibration process. The thermocouple and the flat heater is connected to a PID controller that keeps a con- 


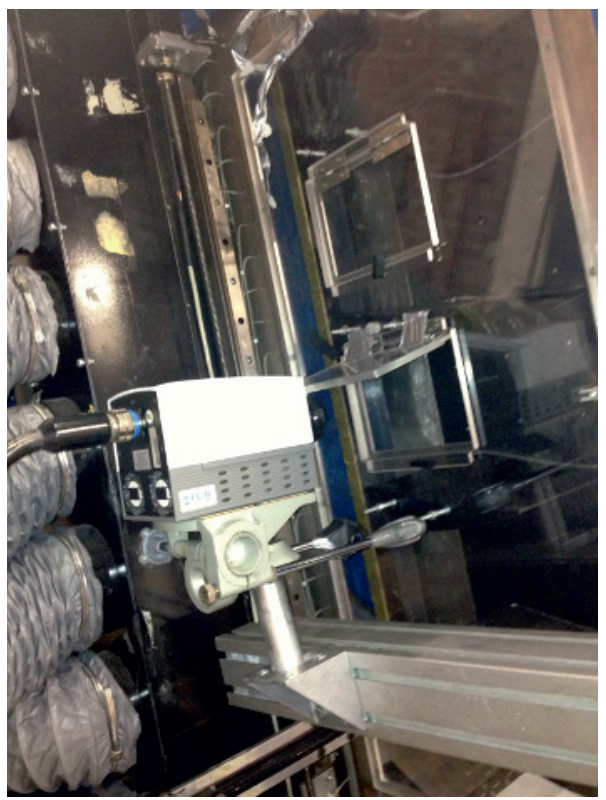

Fig. 5. IR-camera pointing at the instrumented window during the experiments.

trolled constant temperature so that it is possible to provide as many reference temperatures as are needed for the calibration of the camera. The calibration is done by looking at 5 reference temperatures which are in the range of the expected temperatures that are measured during the experiment. For the non-uniformity correction it is needed a cold source (ambient temperature) and a hot source $\left(50^{\circ} \mathrm{C}\right)$. The accuracy of the IR-camera itself is $0.01^{\circ} \mathrm{C}$, but Finally, the accuracy of the IR-camera is about $0.1{ }^{\circ} \mathrm{C}$ due to the fact that the calibration tool has that precision.

Furthermore, it is needed to provide optical access to the camera. On one side of the linear cascade is located the instrumented window for the heat transfer measurement and on the other there is a Plexiglass window which has a low transmissivity in the infrared spectrum that this IRcamera is sensitive to $(3-5 \mu \mathrm{m})$. Therefore, there are several ways to solve this problem. It can be used a special material that has high transmissivity in our range of interest and substitute the Plexiglass by this material (at least in some important locations) or a few small windows can be performed with a fast opening system so that the hot endwall does not feel the flow changes during a big enough period of time so that an IR image can be taken. It is important that the frame rate of this IR-camera is around $60 \mathrm{~Hz}$ during this experiment, but it can be much higher by reducing the camera window size. This frame rate is fast enough to capture the needed amount of pictures and to average them within a period of time where the temperatures keep constant.

\section{Results}

\subsection{On-Design conditions}

For on-design conditions, the flow angle is $25^{\circ}$ and the Reynolds numbers are 300000 and 450000 . Figure 6 shows the results from the 300000 Reynolds number cases. It is important to point out that as it described in section 3 , the
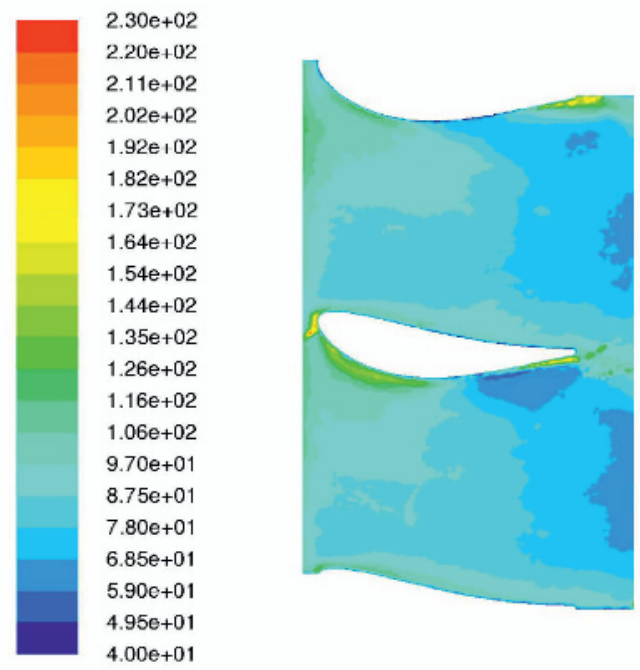

a)

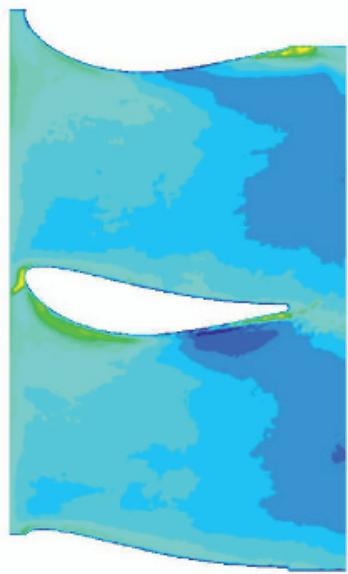

b)

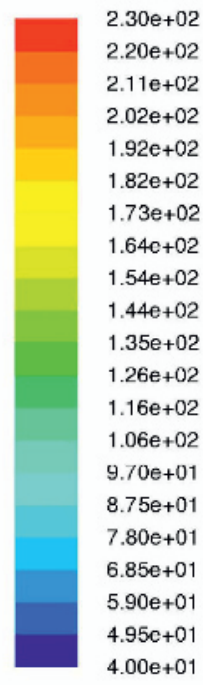

Fig. 6. a) HTC distribution on the endwall without including radiation losses. b) HTC including radiation losses.

radiation heat transfer mechanism influences the results. Especially on the areas where there is higher temperature on the endwall, and therefore where the HTC is lower, the difference between analyzing the results with and without the radiation correction can lead to a $10 \%$ error on the HTC calculation. There can also be seen that there is no separation in this case. Looking at the endwall, in the areas in between the two vanes, the HTC is decreasing in the streamwise direction. This is a similar behaviour as a flat plate case where the flow is fully turbulent. On the suction side of the vane it can be appreciated an area with a very low HTC close to the trailing edge that will change its position for the different cases.

Figure 7 shows the on-design case with a Reynolds number which has been increased to 450000 . There is an expected increase of the HTC in all the areas compared with the previous case due to the fact that the Nusselt number is proportional to the Reynolds, and the HTC is proportional to the Nusselt. In this case there is almost no difference in the HTC distribution on the endwall compared to the lower Reynolds on-design case. The main difference is 

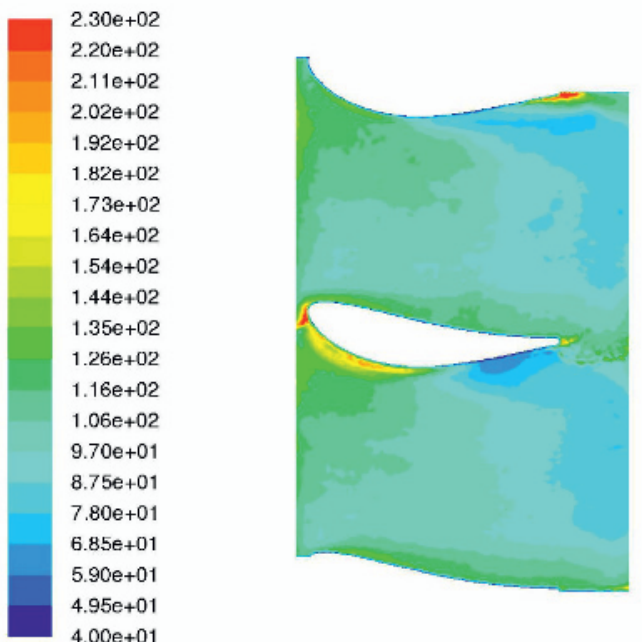

Fig. 7. HTC on-design case with an axial Reynolds number of 450000 .

that the area with a low HTC on the suction side has been displaced downstream.

Furthermore, in both cases, there is a hot spot on the leading edge. There, the HTC achieves its highest value. This area is very important because of the cooling of this area can be critical to avoid structural damage on this component.

\subsection{Off-Design conditions}

Off-design conditions are very interesting because of the risk of flow separation when there are changes on the incident angle to the OGV. The first off-design case is shown in figure 8. The flow incident angle for this off-design case is $40^{\circ}$. It can be clearly seen that there is no flow separation for this case. The flow impinges on the pressure side close to the leading edge and it is generated a vortex that rolls up downstream. Due to the adverse pressure gradient, this vortex is increasing its size. It would be expected to see also the effect of this vortex on the HTC of the vane close to the endwall affecting the flow around this vortex. In addition, comparing on-design case and this case, the flow on the suction side follows almost the same pattern. The area with a low HTC on the suction side, which has been discussed in the previous cases, is displaced upstream.

Figure 9 shows the second off-design case studied. In this case, the incident angle is $-25^{\circ}$. As it can be seen very clearly in figure 9, there is flow separation on the pressure side. There is a separation bubble that decreases the HTC in between the flow and the endwall. Furthermore, the flow is much more turbulent in these cases than in any of the previous cases, which leads to a noisier image. Close to the leading edge, on the suction side of the vane, it is shown a low HTC area where the flow impinges on the vane. From there, there is a vortex that rolls up downstream. From this impingement point, a fraction of the flow moves towards the leading edge to the pressure side and detaches from it. This flow creates a strong line where the HTC is higher than in the separation bubble.
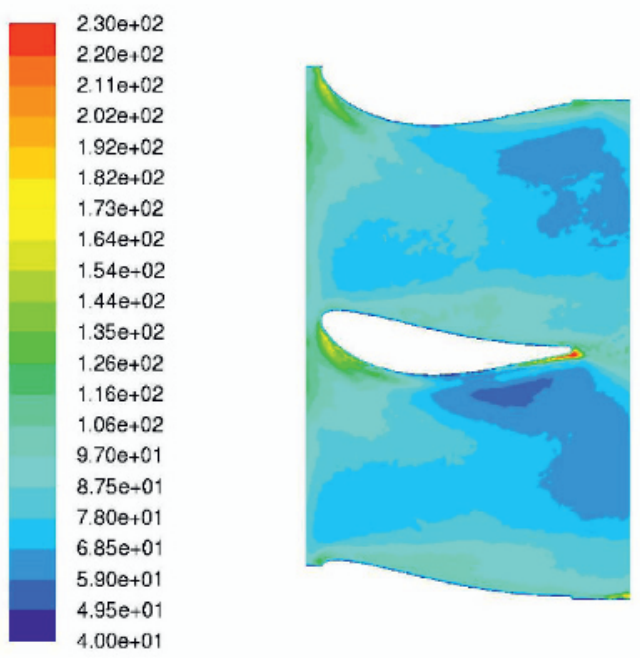

Fig. 8. HTC off-design case $40^{\circ}$.
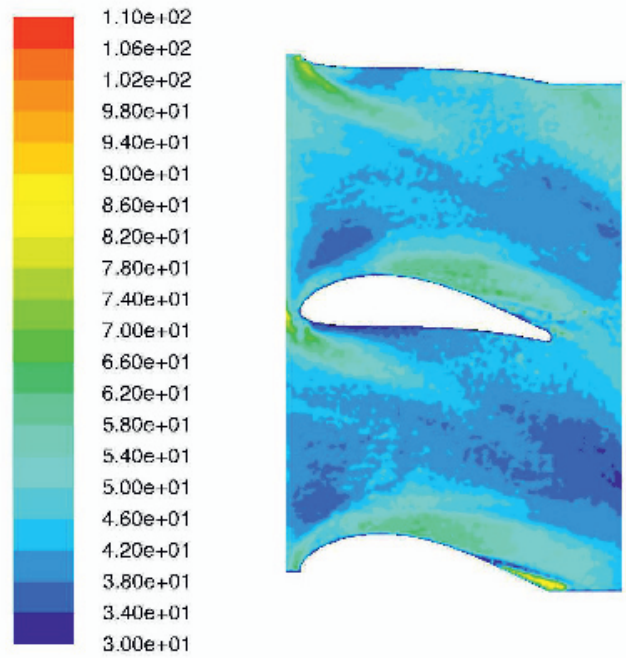

Fig. 9. HTC off-design case $-25^{\circ}$.

\section{Conclusions}

In the present experimental heat transfer study, it has been developed an instrumented window that is designed for measuring heat transfer in a low-speed linear cascade. IR technique has been used for measuring the temperatures needed for the experiment which has shown that the resolution and accuracy of this technique is high. The main drawback is the time spent on having optical access to the endwall. Furthermore, it is important to point out that from our results, the HTC on the upper path is slightly higher than in the lower path, although the general behaviour of the flow is periodic.

In addition, from this experiment, it has been shown that the flow around the vane is very stable in off-design conditions. It has been also detected hot spots close to the leading edge in on-design and off-design cases which are important to study for future application on the cooling of this OGV. Furthermore, in order to understand better all the results obtained from this experiments, it is needed to have detailed experimental information about the flow around the OGVs and the endwall. 
Finally, in the last off-design case $\left(-25^{\circ}\right)$, there is flow separation. It is clearly seen the separation bubble and its consequences on the HTC in the area affected by this phenomena. In addition, in the $40^{\circ}$ off-design case it has been detected a vortex that it is expected to be seen in future studies on the vane (CFD or experimental study).

\section{References}

1. Graziani, R.A., Blair, M.F, Taylor, J.R., and Mayle, R.E., An experimental study of endwall and airfoul surface heat transfer in a large scale turbine blade cascade, ASME J. Engineering for Power, 102 , 257-267 (1980)

2. Goldstein, R.J., and Spores, R.A., 1988, Turbulent transport on the endwall in the region between adjacent turbine blades, ASME J. Heat Transfer, 110 , 862-869 (1988)

3. Wang, H.P., Olson, S.J., Goldstein, R.J., and Eckert, E.R.G., Flow visualization in a linear turbine cascade of high performance turbine blades, ASME J. Turbomachinery, 119, 1-8 (1997)

4. Han, S., and Goldstein, R.J., Influence of blade leading edge geometry on turbine endwall heat (mass) transfer, ASME J. Turbomachinery, 128, 798-813 (2006)

5. Han, S., and Goldstein, R.J., Heat transfer study in a linear turbine cascade using a thermal boundary layer measurement technique, ASME J. Heat Transfer, 129, 13841394 (2007)

6. Han, S., and Goldstein, R.J., The heat/mass transfer analogy for a simulated turbine endwall, Int. J. Heat and Mass Transfer, 51, 3227.3244 (2007)

7. Wang, L., Sundén, B., Chernoray, V., and Abrahamsson, H., Experimental study of endwall heat transfer in a linear cascade, Journal of Physics: Confernce Series 395, 012028 (2012)

8. Wang, L., Sundén, B., Chernoray, V., and Abrahamsson, H., Endwall heat transfer measurements of an outlet guide vane at on and off design conditions, ASME paper GT2013, 95008 (2013)

9. Hjärne, J., Turbine outlet guide vane flows, $\mathrm{PhD}$ thesis, Chalmers University of Technology, (2007)

10. Chernoray, V., Ore, S., and Larsson, J., Effect of geometry deviations on the aerodynamic performance of an outlet guide vane cascade, ASME paper GT2010-22923 (2010)

11. Jimenez, C., Experimental heat transfer studies with infrared camera, Master's Thesis, no. 2013:41, Chalmers University of Technology, Gothenburg, Sweeden (2013)

12. Arroyo, C., Aerothermal investigation of an intermediate turbine duct, $\mathrm{PhD}$ thesis, Chalmers University of Technology, (2009)

13. Rojo, B., Johansson, M., Chernoray, V., and Golubev, M., Experimental Heat Transfer Study in an Intermediate Turbine Duct, 49th AIAA Joint PropulsionConference, AIAA-2013-3622 